\title{
The effects of simplifications on isolated wheel aerodynamics
}

\author{
Sammy Diasinos ${ }^{\mathrm{a}, *}$, Tracie J. Barber ${ }^{\mathrm{b}}$, Graham Doig ${ }^{\mathrm{b}, \mathrm{c}}$ \\ a Macquarie University, Sydney 2109, NSW, Australia \\ ${ }^{\mathrm{b}}$ School of Mechanical and Manufacturing Engineering, The University of New South Wales, Sydney, NSW 2052, Australia \\ c Aerospace Engineering Department, California Polytechnic State University, San Luis Obispo, CA 93407, USA
}

\section{Keywords:}

CFD

Vehicle aerodynamics

Wind tunnel correlation

Wheels

Model simplifications

\begin{abstract}
A B S T R A C T
In order to study the aerodynamic forces and flow features of rotating wheels, compromises and simplifications are often made in wind tunnel testing, and more frequently so in numerical modelling. A CFD approach similar to that commonly used in industry was utilised to investigate common assumptions involving; the influence of geometric fidelity in wheel hub regions, ground representation, the modelling of the contact patch, and the effects of rotation on separation. It was found that the separation and wake characteristics were strongly influenced by the rotation of the wheel; the separation point changed by as much as $90 \%$ compared to a stationary wheel, and drag was close to $20 \%$ less - downforce was approximately $40 \%$ greater. In addition, the modelling of the contact patch, treated here as a small step to facilitate skew-free meshing necessary for a reliable converged result, was seen to cause up to a $52 \%$ difference in predicted lift characteristics, and an increase in the step of just $2 \mathrm{~mm}$ decreased the maximum wake thickness by close to $50 \%$ - considerable changes stemming from superficially-minor simplifications. Including indented wheel hubs proved to be more influential on the production of vortices and wake structures, causing the merging of previously-separate vortex structures. The results point to a need for very careful evaluation of the goals of any study when determining which simplifications can be made in both physical testing and numerical analysis.
\end{abstract}

\section{Introduction}

In studying the effects of flowfields around wheels, simplifications are often applied in order to reduce the complexity of the aerodynamics to a level which can be simulated in a reasonable timeframe using computational fluid dynamics (CFD). In motorsport applications, time-consuming but more realistic-transient analysis is rare due to tight deadlines. In the wind tunnel, the influence of stings, the ability or otherwise to rotate the wheel at all or at a suitable velocity, and the interaction with the ground plane all serve as critical points which can affect the usefulness of the results (Burgin et al. 1986, Lajos et al., 1986). On top of this, satisfactory correlation of wind tunnel and/or CFD results to track data is notoriously difficult and the focus of much time and effort (Cruickshank and Doig 2014).

Publicly-available aerodynamic investigations of an isolated wheel applicable to open wheel racing cars commenced with the three dimensional experimental study undertaken by Morelli (1969). Open-wheel vehicles are used in common types of racing all the way up to Formula 1, with the tyres almost always "slick", in

\footnotetext{
* Corresponding author.

E-mail address: sammy.diasinos@mq.edu.au (S. Diasinos).
}

that they rarely feature a tread pattern. Category rules dictate that the wheels not be shrouded or enclosed, thus fully exposing them to the oncoming flow. The wheel wakes therefore have a very strong bearing on the aerodynamic performance of other vital components (Diasinos et al., 2014).

In Morelli's study, the ground was represented by a stationary ground board which included a recess for the wheel to rotate within (i.e. the wheel was not in contact with the surface), which resulted in a cumulative downforce-this was later shown to be purely a product of the ground representation by Cogotti (1983), who demonstrated that an isolated wheel in contact with a true ground should produce lift. This was re-enforced by the experiments of Stapleford and Carr (1970), who concluded that the only way of making certain that the flow features reproduced during an experiment are representative of that of a wheel belonging to an open wheeler racing car is to have a rotating wheel in contact with a moving ground.

Fackrell and Harvey (1973, 1975) and Fackrell (1975) experimental investigations for three wheel widths and two wheel shoulder shapes would become the benchmark for later research undertaken on exposed wheels, although information about the sting geometry and other influential parameters was not detailed. Lift and drag were determined to be approximately $42 \%$ and $25 \%$ lower respectively than experienced by a stationary wheel in 
contact with a stationary ground. Between these two scenarios, the wheel rotation moved the forward stagnation point approximately $15^{\circ}$ down towards the front contact patch at the ground. A peak pressure forward of the contact patch greater than two was obtained, due to the moving boundaries transmitting energy into the flow through the shear stresses created by the boundary layers that form over the two converging surfaces. This increase in pressure achieved at the wheel centre was expected to cause a jetting action at either side of the front contact patch to increase in strength and to encourage the formation of two stronger vortices from each side of the wheel. These features are shown schematically in Fig. 1 to orient the reader to the flowfields shown in later sections.

The first non-intrusive wake measurements of an isolated wheel were made by Knowles et al. (2002) using Laser Doppler Anemometry and a rotating wheel with a moving ground. Only four vortex structures could be found in the wake of the wheel, with two counter rotating vortices forming at the base of the wheel wake adjacent to the floor as previously measured by Bearman et al. (1998).

Reynolds-Averaged Navier Stokes (RANS) computational models specifically of wheels emerged in the late 1990's: Axon et al. investigated an isolated wheel using a commercial CFD code (FLUENT). The wheel geometry utilised shared a common wheel width to diameter ratio as that used by Fackrell (1975) but was

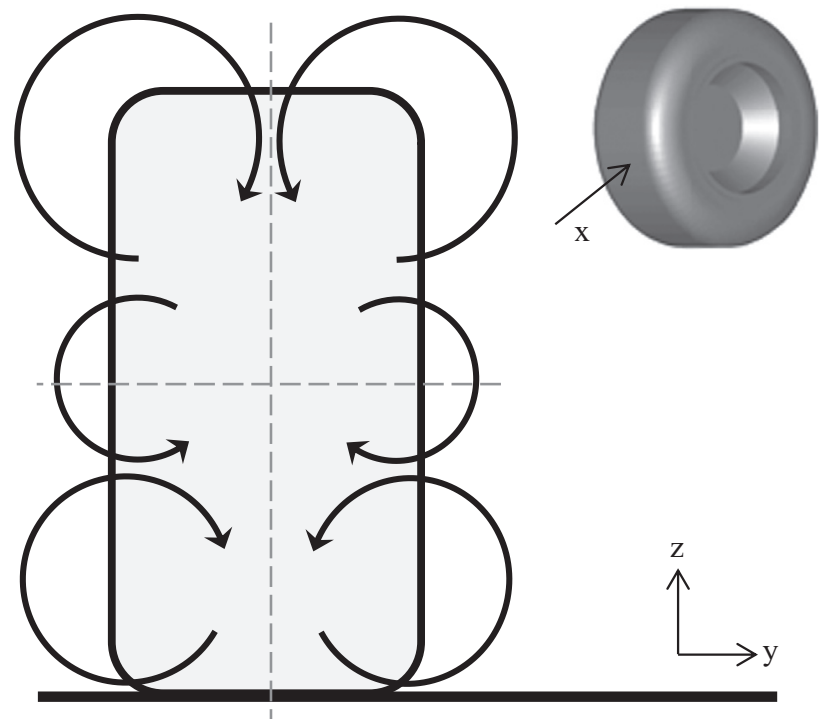

Fig. 1. Schematic of main vortices produced by a wheel, viewing on a plane normal to the freestream. simplified such that the wheel hubs were removed and the wheel shoulder was replaced with a constant radius (as in Fig. 2c). The model indicated that the rotating wheel produced $C_{\mathrm{P}}$ values greater than 2 in the region forward of the contact patch and confirm the hypothesis that the jetting action forward of the wheel assists the formation of the vortical structures emanating from either side of the wheel (Fackrell and Harvey, 1973). Axon et al. (1998) also highlighted the difficulty associated with creating a mesh around the contact patch of the wheel. By meshing the acute junction between the ground and the wheel surfaces, a large number of skewed control volumes were introduced and so a small step around the perimeter of the contact patch was introduced. Unfortunately the effect that this common simplification might have on the flow structures was not investigated at the time.

McManus and Zhang (2006) conducted a transient computational analysis, using unsteady RANS, of an isolated wheel, although reported time-averaged results. While it is inarguable that flow around a wheel is inherently unsteady (Pirozzoli et al., 2012; Dassanayake et al., 2012), from an industrial point of view the computational power and runtimes involved remain largely impractical for anything other than steady RANS despite the potential for improved accuracy. Similarly, studies investigating flow around well-defined, high fidelity models featuring tyre treads, hub spokes, brake ducts, tyre camber and deformation, etc., lead to problems in reproducing the intricacies of the experiment with confidence in CFD, as many features are difficult to resolve in the model but strongly influence the wake (Saddington et al., 2007; Issakhanian et al., 2010; Axerio-Cilies and Iaccarino, 2012).

In this vein, the present study aims to investigate ways in which tunnel and numerical data sets can feature better correlation through modelling choices which should be considered from the start of a campaign featuring one approach or both. The results focus on the effects of how the wheel/ground contact patch is modelled, the importance of wheel rotation and defined separation points, and the role of the wheel hubs in influencing the force and wake characteristics.

\section{Numerical method}

All original results presented here for the asymmetric Fackrell A2 wheel-the dimensions of which are shown in Fig. 2. (along with variants tested for discussion in the subsequent sections)-were computed using ANSYS Fluent using the segregated pressurebased solver, second order upwinding for discretized terms, and a standard SIMPLEC algorithm for pressure-velocity coupling.

This study focused on steady-state RANS solutions, as this remains by far the most common approach in automotive and

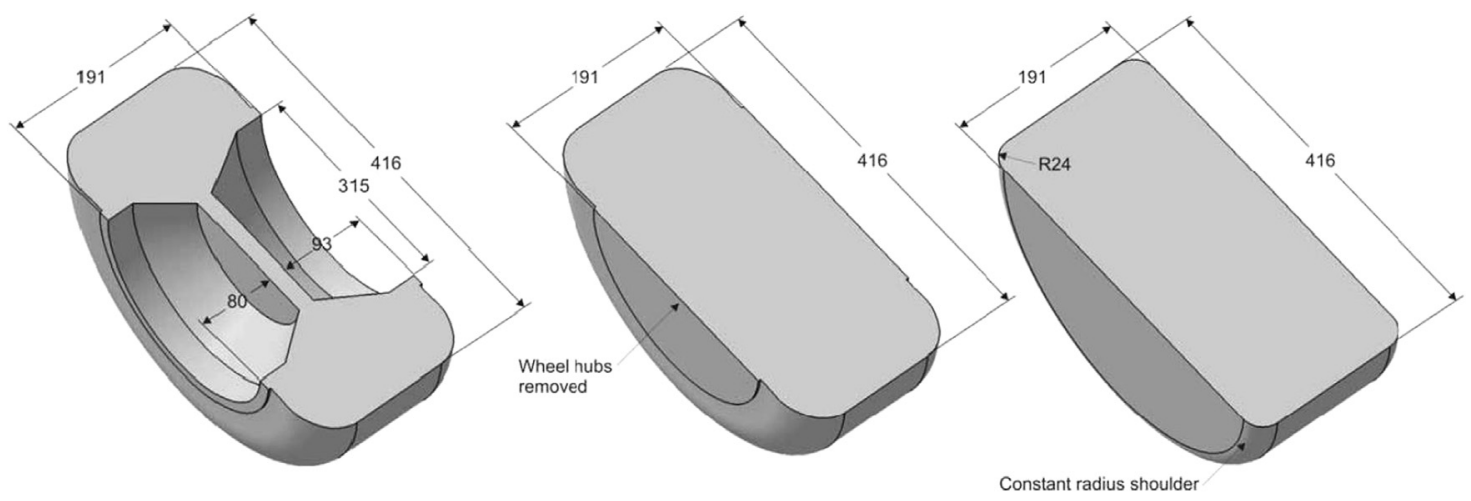

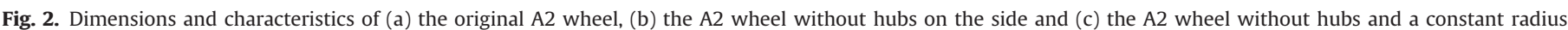
shoulder (W1 wheel). 
autosport applications (including comparisons to time-averaged wind tunnel data), though it is acknowledged that the flow would exhibit unsteady characteristics in real life which may further exaggerate the influence of simplifications made to the wheel representation. For this reason, additional simulations were run as transient (unsteady RANS) and the flowfields were time-averaged in order to assess the suitability of steady-state simulations for the present investigation. Because of a similar industry standard, all simulations were run as incompressible, although local density changes, particularly in the contact patch region, may have a slight influence on the flow (Doig et al., 2011; Doig, 2014; Keogh et al. 2014).

Convergence was deemed acceptable for steady-state solutions when the residual errors dropped below a normalised level of $10 \mathrm{e}^{-4}$, which typically was achieved after 2000 iterations. Furthermore, the simulation was only deemed complete when the aerodynamic coefficients ceased to change by more than $0.1 \%$ over a continued further 1000-2000 iterations, indicating the solution had reached an established level which would not be altered or improved for that particular mesh and turbulence model.

Two transient comparison simulations were run at timesteps of $0.00025 \mathrm{~s}$ and $0.0005 \mathrm{~s}$, which were calculated to be sufficient to account for flow movement through a single cell in one timestep based on the cell-to-velocity ratios existing in the smaller-volume cells - maximum CFL was 0.95 in the vicinity of the wheel. The simulations were initiated using the steady-state solution as a starting point, after which $2 \mathrm{~s}$ of real-time was allowed to pass in order for the transient flowfield to fully-establish before data was taken.

Hybrid meshes were constructed featuring fully-structured hexahedral mesh in the vast majority of the domain, with semistructured (swept) tetrahedral mesh in the region of the contact patch to avoid excessive cell-stretching in highly-acute angles. An image indicating the domain size and an example mesh is shown in Fig. $3 a$ and $c$. The wall $\mathrm{y}^{+}$values in regions of attached flow were below 1, enabling appropriate resolution of the boundary layers and the use of the enhanced wall model available for the Realizable $k-\varepsilon$ turbulence model-the near-wall mesh was kept constant in mesh refinement studies for consistency in wall model usage. For initial comparisons, the contact patch between wheel and ground was modelled as a "step" of 0.0028 of the wheel diameter-the influence of this is examined later in the manuscript. Fig. 3d indicates the procedure to create this step - first the wheel is lowered where the ground boundary would be, to create an intersection where previously the surfaces met at a tangent. Then the wheel is raised above the ground by that amount and a plinthlike surface is extruded from the new bottom surface to the ground. This creates a region that can be satisfactorily meshed without inducing excessive skew, and allows a sufficient number of near-wall cells to be clustered into this crucial region; the step had 70 nodes to define it in the vertical sense after it was found that the high pressure peak was exceptionally sensitive to this region, and coarser meshes were unable to approach the experimental values; detail of this mesh feature is included in the inset of Fig. 3a.

\subsection{Comparison to experimental study of Fackrell}

\subsubsection{Boundary conditions and notes on comparison data}

Validation of the computational model of the A2 Fackrell wheel was conducted against the original experiments; the boundary conditions of the wind tunnel were reproduced as accurately as possible, both in terms of the dimensions reported in Fig. 3. (including a region upstream modelled as a slip wall to account for the location of the start of the moving ground closer to the wheel) and the oncoming flow speed of $18.6 \mathrm{~ms}^{-1}$ with corresponding wheel rotational velocity of 89.43 rads $^{-1}$. Full details may be found in literature (Fackrell and Harvey, 1973; Fackrell, 1975). The blockage of the wheel model in the test section is $4.1 \%$ based on projected frontal area. The inlet turbulence intensity, I, was set at $0.2 \%$, with an appropriate length scale used to calculate values of $\mathrm{k}$ and $\varepsilon$ as $0.0021 \mathrm{~m}^{2} / \mathrm{s}^{2}$ and $0.00018 \mathrm{~m}^{2} / \mathrm{s}^{3}$ respectively.

The experimental lift and drag results were obtained through integration of the measured static pressure recorded from 25 pressure tappings distributed across the tread, wheel shoulders and inner hubs of the wheel as it rotated. This method was extrapolated to determine the lift and drag coefficient of the wheel within an estimated accuracy of $\pm 10 \%$, however the method is highly approximate and does not account for the interference of the sting and balance arms on one side of the wheel (which is also why the wheel is not perfectly symmetric); for this reason the force results are not treated as being accurate enough for valid model comparisons. Only the measured pressure distributions, both on the wheel centre circumference and in the wake, are used for comparison.

\subsubsection{Mesh resolution}

A grid convergence study was conducted in which successively finer meshes were applied. The coarse mesh featured a total of 2.03 million cells, with 270 around the circumference of the wheel. The finest mesh was a reduction of cell size in all areas (i.e. including all off-surface regions), resulting in a total of 8.06 million cells with 370 around the circumference of the wheel. Both lift and drag were used as verification parameters in determining gridindependence of the results, and the coefficients obtained are plotted in Fig. 4. Both parameters exhibit relatively little change

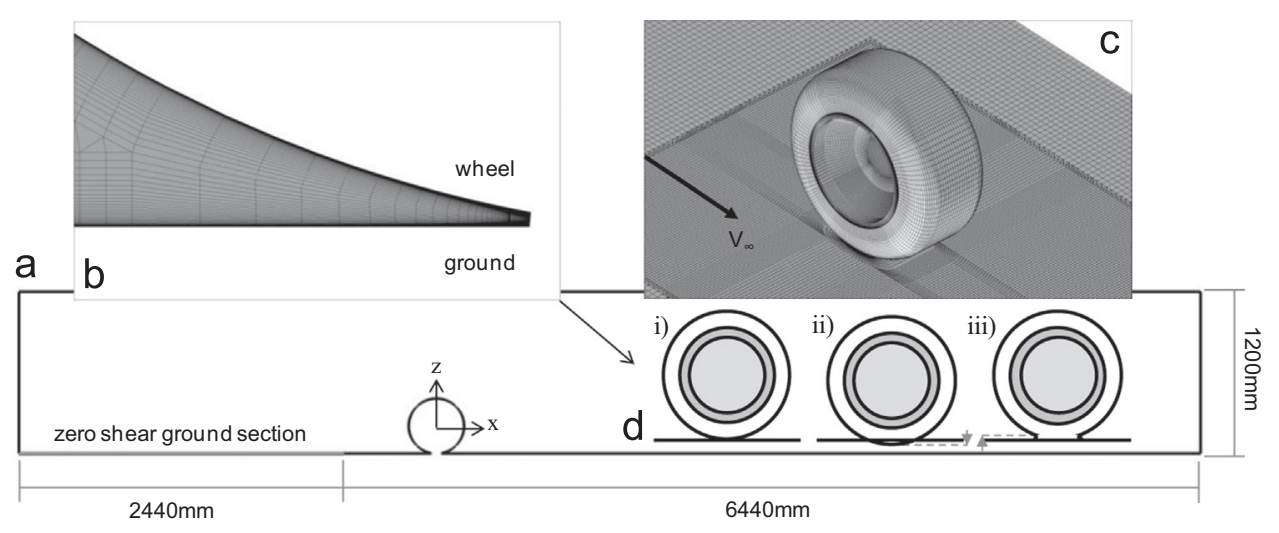

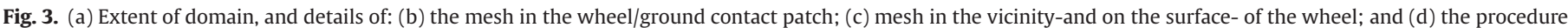
for creating the contact patch. 


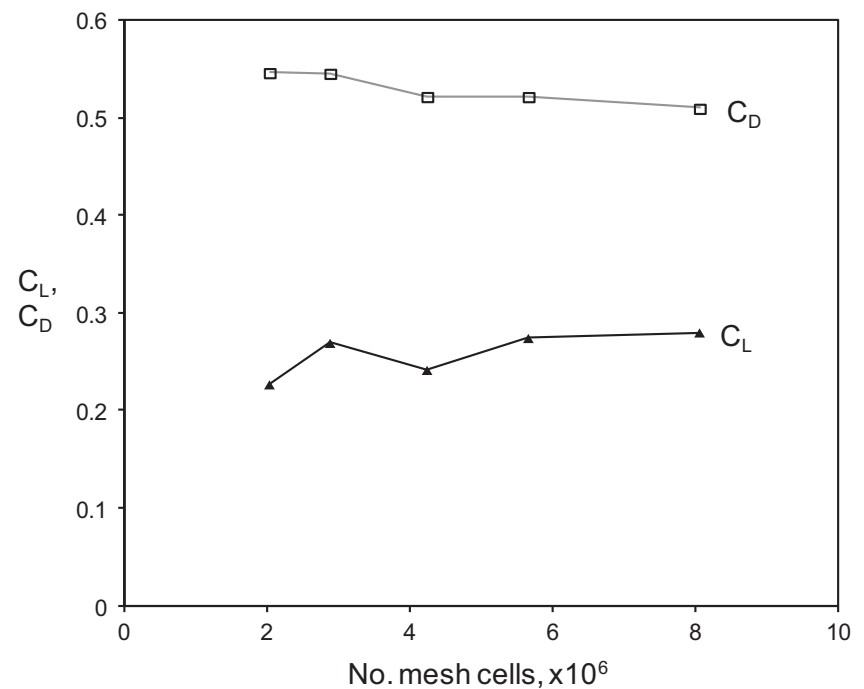

Fig. 4. Lift and drag coefficients with increasing mesh resolution.

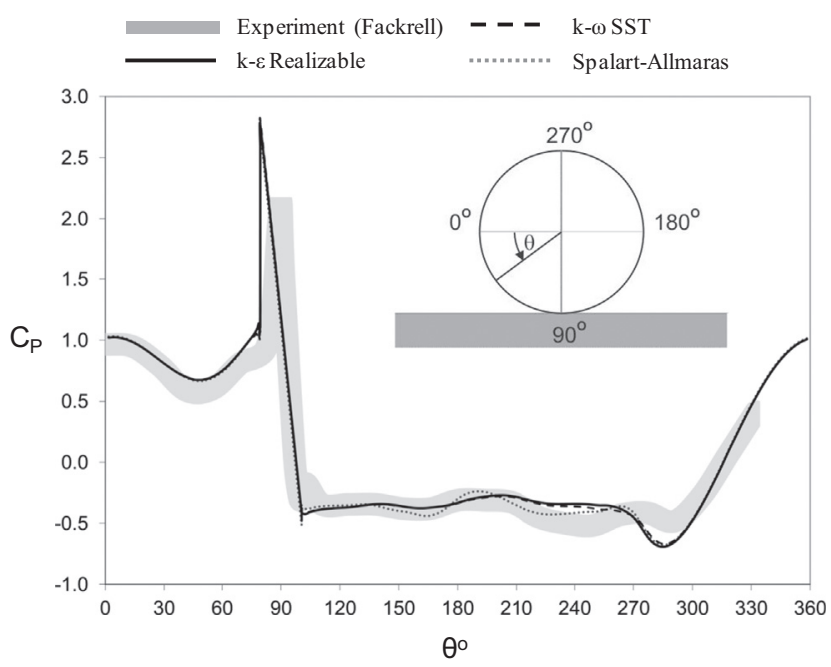

Fig. 5. Centre circumference pressure coefficient distribution for various turbulence models.

( $<2 \%$ difference in $C_{\mathrm{D}}$ between 5.6 million cells and 8.06 million, and $<2.5 \%$ difference in $C_{\mathrm{L}}$ ), indicating that further refinement would not bring significant additional accuracy that would outweigh the computational cost of further refinement. As a result the $\sim 8$ million mesh was used for all subsequent simulations.

\subsubsection{Turbulence modelling}

Three commonly-used turbulence models were tested for effectiveness in reproducing the experimental pressure distributions, the 1-equation model of Spalart and Allmaras (1992) (SA), Menter's SST variant of the $k-\omega$ model (Menter, 1994), and the Realizable $k-\varepsilon$ model (Shih et al., 1995). The latter have been recently applied to similar flowfields, with the Realizable model proving more accurate in determining the vortex locations and strengths when compared to experimental data (McManus and Zhang, 2006). Comparisons to the centre circumference pressure distributions of the rotating A2 wheel, illustrated in Fig. 5, indicated that all models reproduced the features well, with the SST and Realizable models yielding almost identical plots. While there is little to indicate any major discrepancy on behalf of the SA model in this prediction, the reported deficiencies of the model when applied to strongly-separated flows meant that the
Realizable model was deemed preferable, further supported by other literature (McManus and Zhang, 2006). In general, the pressure distributions indicate that all models are able to reproduce the experimental data reasonably well within the approximated margin of error from the wind tunnel tests, with the notable exception of the separation behaviour at approximately $280^{\circ}$, just past the uppermost point of the wheel.

The model indicates a swift recovery to a value closer to the freestream, as do results presented by McManus (2006) for the same portion of a rotating wheel, which may suggest that this discrepancy may be due to an error or uncertainty associated with the experiment, or perhaps the inadequacy of RANS modelling in describing the flow here. One would expect this separation feature to be not only most sensitive to mesh and turbulence model, but also to any upstream influence; in the latter case, the high pressure spike at the contact patch, somewhat over-predicted in the CFD, would exert a lingering influence over the rest of the pressure gradient around the wheel including the location of the forward stagnation point-a primary reason why the modelling of the contact patch was investigated for the present study.

\subsubsection{Additional Comparisons to experiment}

Fackrell and Harvey (1973) also took off-surface total pressure measurements at planes located $0.10 d, 0.35 d$ and $0.52 d$ downstream of the wheel centre to determine the wake structure behind the rotating wheel. This was only conducted for half of the wheel wake as the opposite half was expected to be affected by the presence of the wheel struts (thus interference effects were not properly quantified). The 0.9 total pressure coefficient contour line was presented as this was expected to be indicative of the wake's extremities. In Fig. 6, the results obtained using the present computational model have been presented as flooded contours with the $C_{\mathrm{PT}}$ value of 0.9 indicated by a solid black line. The results obtained experimentally by Fackrell are overlaid using a black dashed line.

The total pressure coefficient comparison suggests that the separation point for the rotating wheel over the wheel tread, or centre circumference, has been predicted with enough accuracy to produce a realistic wake structure. Similarly, the wheel wake width also shows acceptable agreement; some variations occur where a vortex is formed in the wheel hub and the lower wake region where one of the two main wheel vortices are expected to form $(z / d=0.7)$.

\subsubsection{Assessment of steady state assumption}

While the steady state assumption is necessarily used in industry, it is acknowledged that unsteady flow features are influential for the aerodynamics of such a bluff body. A series of unsteady RANS runs were conducted to investigate whether any major flow feature differences would emerge from a time-averaged compilation of results. Two time steps (0.0005 and 0.00025$)$ were implemented, with the results proving near-identical when averaged over a timeframe of $0.7 \mathrm{~s}$; thus the $0.0005 \mathrm{~s}$ outcomes are discussed here.

Fig. 7 shows the wheel centre pressure coefficients between the steady and unsteady runs as compared to the experiments in literature. The only distinguishing variances come over the top of the wheel in the thickening of the boundary layer and eventual separation - the exact mean separation location is different by around 2 degrees, and neither simulation is especially effective at capturing the experimental behaviour as previously discussed. The peak low pressures produced after the separation point are similar, with the unsteady case averaging a slightly less pronounced value - the green shade indicates the standard deviation of values from the unsteady run, indicating that at the maximum separation point in the cycle, the pressure coefficient drops considerably 

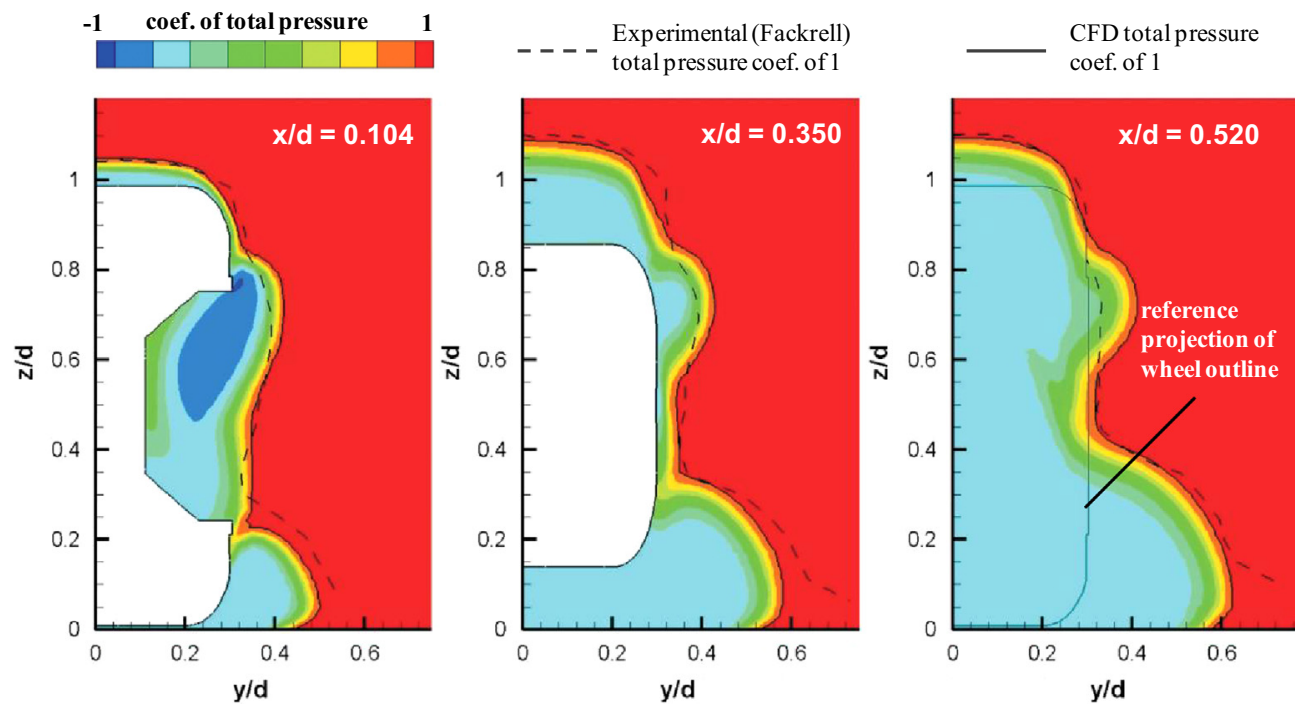

Fig. 6. Contours of coefficient of total pressure at planes normal to the freestream in comparison to experiment.

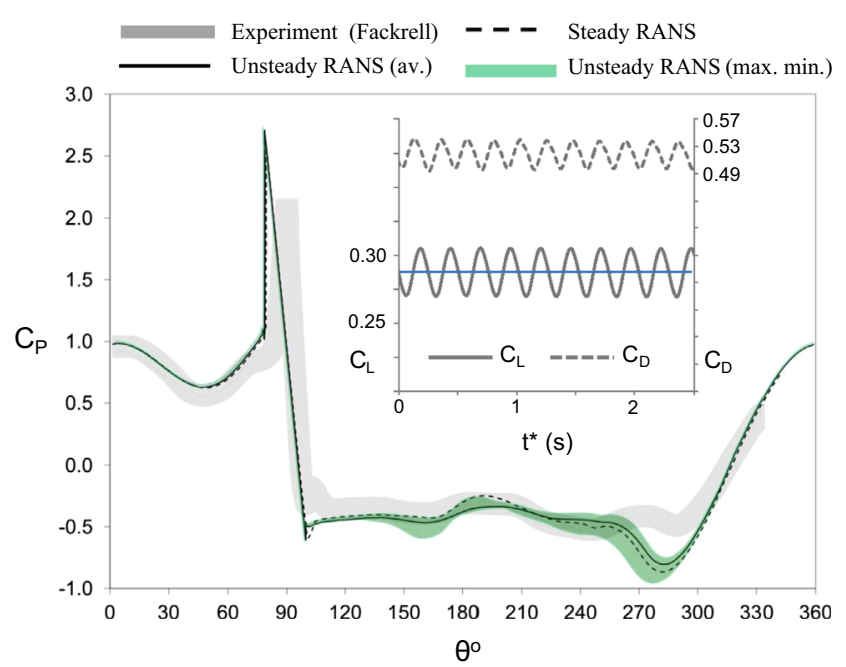

Fig. 7. Centre circumference pressure distribution for steady state vs. unsteady (averaged) results, and (inset) sample transient $C_{\mathrm{L}}$ and $C_{\mathrm{D}}$ values over a typical $2.5 \mathrm{~s}$ period.

below the steady state prediction to almost -1 . However, for the majority of the timeframe the flow remains attached towards the furthest extent, at around $270^{\circ}$. The inset graph of Fig. 7 (force coefficients) indicates that the unsteady results exhibited regular vortex shedding induced by the oscillating upper separation point, however the magnitude of variation and the notable consistency highlights the diffusivity of the RANS approach in general; the variations in coefficients are too regular and narrow in range to be truly typical of what could be expected in reality or from an equivalent LES or DES simulation. Nevertheless the validity of the steady-state RANS approach over averaged unsteady RANS is satisfactory as the mean outcomes are near-identical.

A further indication of the similarity between the two types of simulation is seen in Fig. 8, where total pressure coefficient values on perpendicular planes close to the middle of the wheel at $x=0.104 d$ and further downstream at $0.350 d$ are plotted. There are very subtle differences in the lateral extent of the averaged pressure field which "pumps" out of the hub region, and the tyre "squish" from flow being pushed out of the contact patch zone is slightly affected. In addition, as is clearer at $x=0.350 d$, the slightly earlier average separation point creates a slightly taller wake (as seen in the contours extending above $z / d=1$ ), though one which is generally equivalent in strength and structure to the steady state result. Due to these similarities, continued transient runs were not deemed necessary in order to productively examine the particular influences that form the focus of the main investigation.

\section{Effect of wheel rotation and separation on force and wake characteristics}

The initial hypothesis proposed by Fackrell (1975) to explain the large variation in the primary wheel vortex position between rotating (with moving ground) and stationary (with stationary ground) models suggested that a pair of additional vortices form forward of the rotating wheel, due to the flow separating from the ground. It was believed that these vortices alter the path of the oncoming flow around the front contact patch allowing the primary wheel vortices to form in a more central and higher position within the wake of a rotating wheel. The computational results obtained thus far have provided no evidence of these vortices, in agreement with other reports (McManus and Zhang, 2006).

An alternative theory proposes that the cause of the wider and lower position of the primary wheel vortices associated with a stationary wheel is due to the ejected flow from the front contact patch being deflected to a lesser extent by the oncoming flow due to the boundary layer formation over the stationary ground (McManus and Zhang, 2006). As a result, the jetting action was expected to contribute a greater cross component in the stationary wheel wake than that which would be experienced by the rotating wheel with a moving ground.

To examine this, the stationary ground was replaced with a slip condition so that the boundary layer would be completely eliminated, and the stationary wheel was used. A moving ground condition was not used since a boundary layer, albeit smaller, would still develop (Barber et al., 2002; Kim and Geropp, 1998). According to the hypothesis, a wheel wake structure that contains the primary vortices in a position that more closely resembles that of a rotating wheel should be obtained since the ejected flow can now be deflected to the full extent of the oncoming free stream velocity.

Total pressure contours and in-plane vectors located at $x / c=0.64$ were plotted with the wheel outline shown in grey for reference (Fig. 9). The results indicate that replacing the stationary 

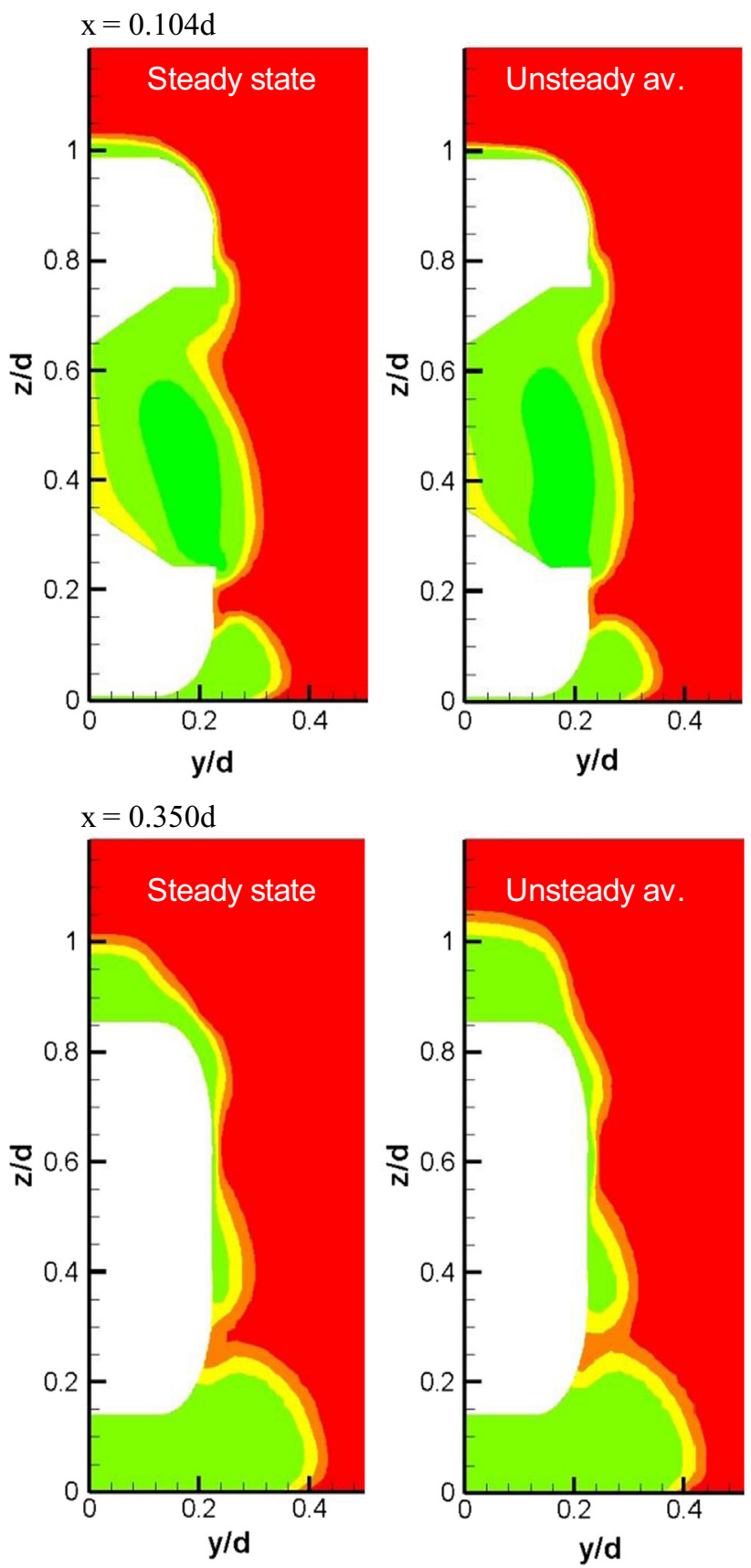

\section{$\mathrm{CP}_{\mathrm{T}}$}

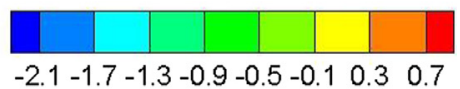

Fig. 8. Total pressure coefficient contours on planes bisecting the wheel close to the centre and towards the rear, for unsteady vs. steady state results.

ground with a slip condition does not alter the lower wheel wake in any significant manner. Despite the wheel wake taking a more asymmetric shape, the primary wheel vortices have formed in a similar position (Fig. $7 b, y / d=-0.2, z / d=0.2$ and $y / d=0.15, z /$ $d=0.15$ ) compared to stationary wheel results (Fig. $7 c, y / d=-0.2$, $z / d=0.15$ and $y / d=0.15, z / d=0.2)$. The lift and drag values also closely resemble those of a stationary wheel, further confirming that no significant variation has been obtained (Table 1 ). Therefore the influence that the boundary layer developing over a stationary ground has on the jetting action can be largely eliminated as an explanation for the variation in the lower wheel wake shape between a stationary and rotating wheel.
Further to the increased jetting action forward of the front contact patch, Fackrell also indicated that the wheel rotation causes an earlier separation point from the top wheel tread. Isolating these two characteristics of a rotating wheel in the A2 CFD model was achieved by splitting the wheel boundary into upper and lower boundaries and then only applying the wheel rotation to either boundary. In the case where the lower wheel region was rotated, a moving ground was also employed. As demonstrated in the inset in Fig. 10, the lower wheel boundary included the region of the wheel between $-45^{\circ}<\theta<135^{\circ}$ while the remaining portion of the wheel was included in the upper wheel boundary.

This unorthodox approach successfully managed to independently create the main features associated with a rotating wheel. The ability to apply such a boundary condition with ease also demonstrates one advantage of using a computational model for such an investigation in comparison to taking a purely experimental approach. By applying the wheel rotation at the top, an earlier separation point that closely resembles that of the rotating wheel has been achieved while at the base, the y-velocity components indicates that no additional jetting action has been produced The opposite holds when the wheel rotation is applied only to the bottom portion of the wheel, with the separation being representative of the stationary case while the jetting action experienced is indicative of a rotating wheel.

The circumferential pressure coefficients (Fig. 10) also confirm that the flow features located at the base or at the top of a rotating wheel have been reproduced and that the two characteristics associated with the wheel rotation have been created independently. The high pressure coefficient peak of the rotating wheel, which is responsible for the increased jetting action, was replicated when only the bottom portion of the wheel rotated. This has had no effect on the separation point from the top of the wheel with pressure coefficients in the region of $190^{\circ}<\theta<360^{\circ}$ of the wheel being identical to those associated with a stationary case.

To determine the influence of wheel rotation on the vortices within the wake structure, total pressure contours and in-plane velocity vectors were used to compare the wake on the $x / c=0.75$ plane (Fig. 9). Based upon these results, the separation point from the top wheel tread appears to be the critical flow feature that causes the primary wheel vortices to form in a higher and more inboard position (Fig. 11a, $y / d=-0.1, z / d=0.45$ and $y / d=0.15, z$ | $d=0.4$ ). The top-rotating wheel also reproduced the upper wheel vortices associated with the wheel tread separation (Fig. 11b, $y /$ $d=-0.1, z / d=0.75$ and $y / d=0.1, z / d=0.75)$. When only the top portion of the wheel boundary was rotated, the primary wheel vortices were located more inboard and higher than those associated with a completely rotating wheel (Fig. 11b, $y / d=-0.15, z$ | $d=0.3$ and $y / d=0.15, z / d=0.3$ ) suggesting that the jetting action draws the primary wheel vortices outboard and lower.

This also indicates that the wheel tread separation point controls the distribution of flow entrainment into the wheel wake from either the top of the wheel or around the sides and is therefore independent of the extent of the jetting action forward of the wheel. As the separation point from the top surface of the wheel moves forward, less flow is entrained from the top of the wheel and this is compensated by an increased entrainment from the sides of the wheel. For this reason the rotating wheel lacks the large downwash component in the central wake region observed for the stationary wheel (Fig. 11d). The main vortices for the stationary wheel are forced down and apart by this downwash component and therefore form further from the central plane and closer to the ground causing the wider lower wheel wake.

Therefore, while the jetting action may make a small contribution to the final position of the primary wheel vortices, when the top wheel tread separation is delayed, the downwash in the wheel wake becomes the dominant feature in determining the 
a

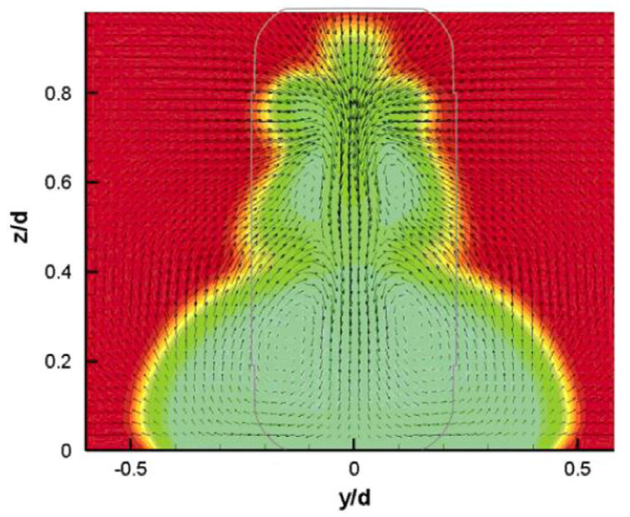

C

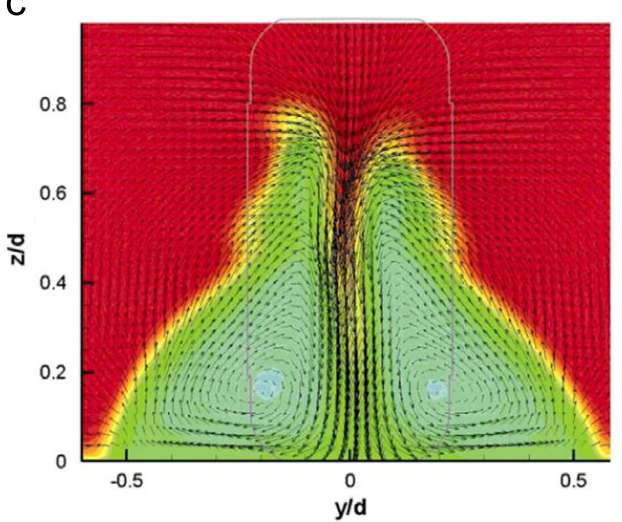

b

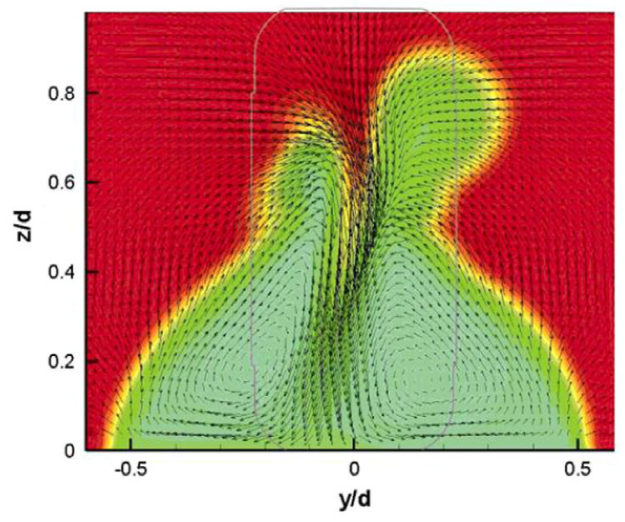

$-1 \quad$ coefficient of total pressure 1

Table 1

$\mathrm{C}_{\mathrm{L}}$ and $\mathrm{C}_{\mathrm{D}}$ for different boundary types

\begin{tabular}{|l|l|l|l|}
\hline Coef. & $\begin{array}{l}\text { Rotating } \\
\text { A2 }\end{array}$ & $\begin{array}{l}\text { Rotating } \\
\text { A2 (slip } \\
\text { ground) }\end{array}$ & $\begin{array}{l}\text { Stationary } \\
\text { A2 }\end{array}$ \\
\hline $\mathrm{C}_{\mathrm{L}}$ & 0.28 & 0.43 & 0.43 \\
\hline $\mathrm{C}_{\mathrm{D}}$ & 0.51 & 0.62 & 0.60 \\
\hline
\end{tabular}

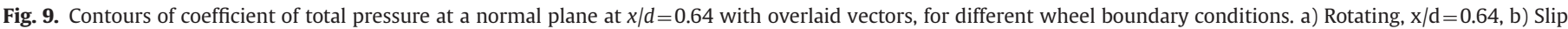
ground, $x / d=0.64$ and c) Stationary, $x / d=0.64$,

Table 1

$C_{\mathrm{L}}$ and $C_{\mathrm{D}}$ for different boundary types.

\begin{tabular}{llll}
\hline Coef. & Rotating A2 & Rotating A2 (slip ground) & Stationary A2 \\
\hline$C_{\mathrm{L}}$ & 0.28 & 0.43 & 0.43 \\
$C_{\mathrm{D}}$ & 0.51 & 0.62 & 0.60 \\
\hline
\end{tabular}

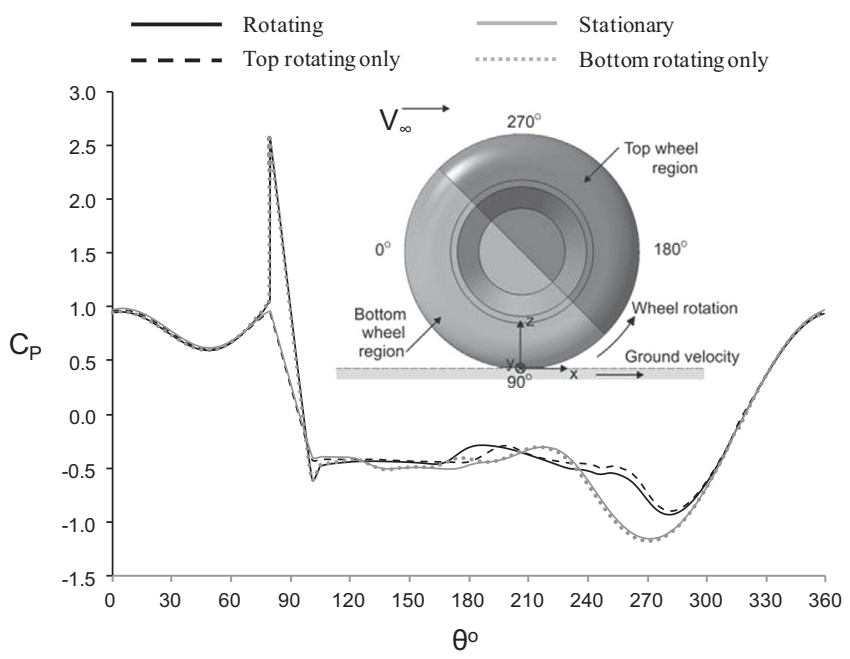

Fig. 10. Centre circumference pressure coefficient distribution for different wheel boundary conditions.

final position of the primary wheel vortices. Based on this, if it were possible to deliberately separate the flow from the wheel tread of a stationary wheel in a comparable position as that experienced by a rotating wheel, it would be expected that a similar wake structure to a rotating wheel will be obtained without the wheel rotation or moving ground being employed. Such a solution would be particularly useful for experimental research in facilities that do not have a moving ground and/or where the wheel cannot be rotated.

\section{Variations due to size of the contact patch}

A difficult aspect of numerical modelling is the accurate representation of the contact patch between the wheel and the ground. This arises in part because the wheel tread is theoretically tangent to the ground for a solid wheel and meshing this geometry will lead to highly skewed cells around the contact line. This effect is further exacerbated by the number of cells required to maintain an appropriately low $\mathrm{y}^{+}$value and to capture the pressure spike resulting from the converging surfaces. For the present study, the geometry of the wheel was modified to include a small face around the wheel's contact patch that subtended an equal angle to the ground and the wheel tangent. Axon et al. (1998) also utilised a step around the contact patch during his numerical study, but other authors conducting numerical investigations have not explicitly indicated how this problem has been approached or overcome.

All results presented to this point have included a step height of $0.0028 d$. This was initially chosen as it was the smallest step that could be achieved without adversely affecting the quality of the mesh surrounding the contact patch. Varying the size of the contact patch by increasing the height of the step to a maximum of $0.0085 d$ (the height of the top of the wheel remaining unchanged) also gives some insight into the validity of using a solid wheel during wind tunnel testing, as is common, to emulate the real 
a

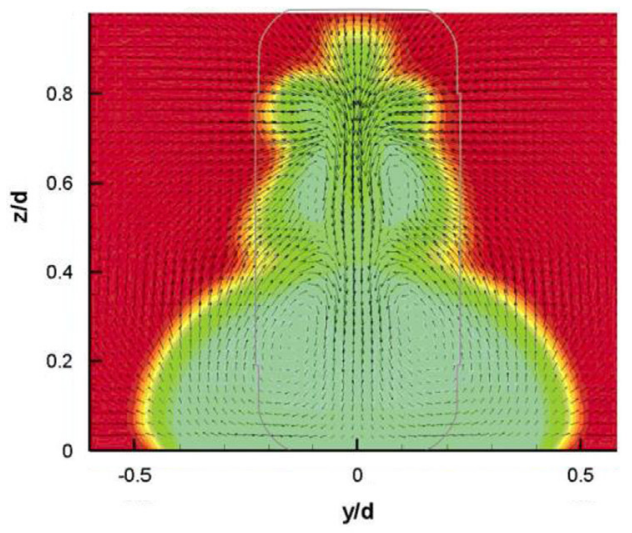

C

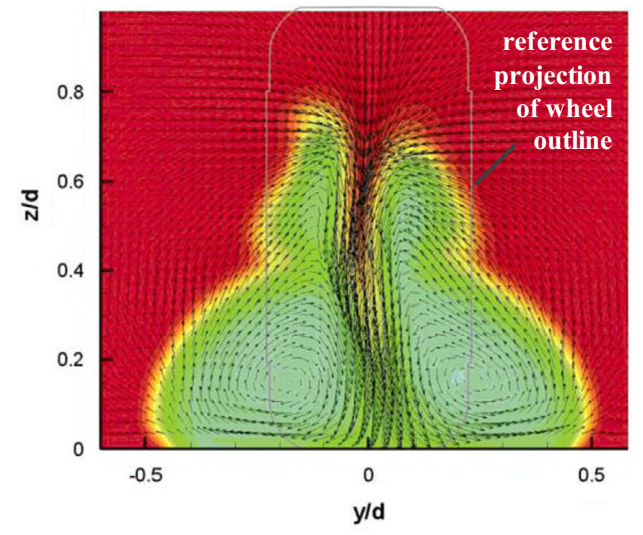

b

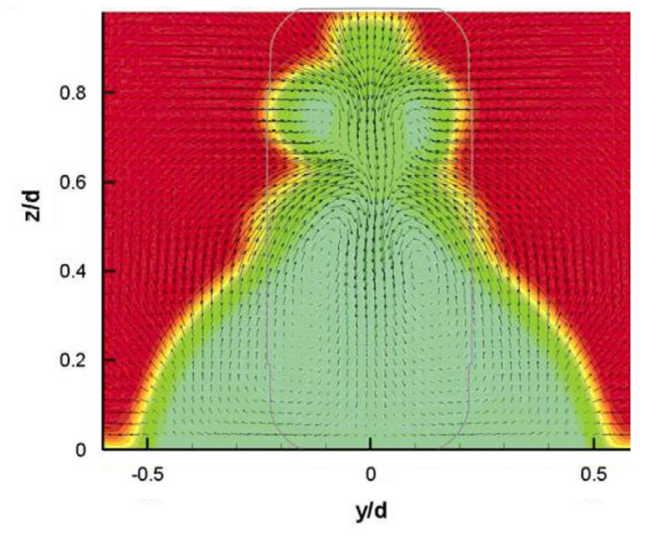

d

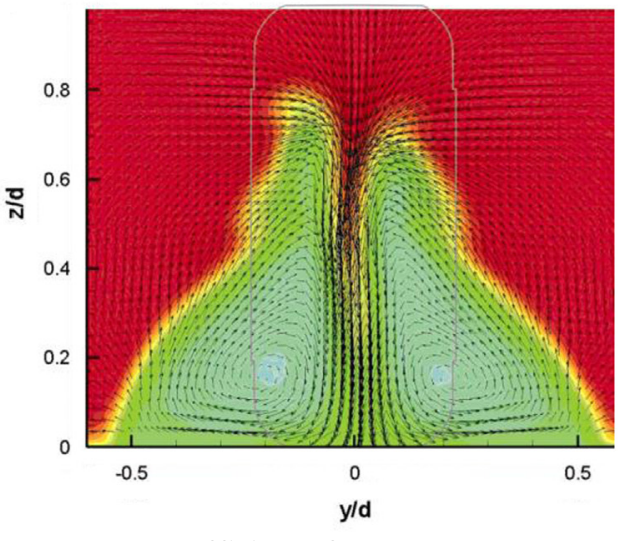

$-1 \quad$ coefficient of total pressure $\quad 1$

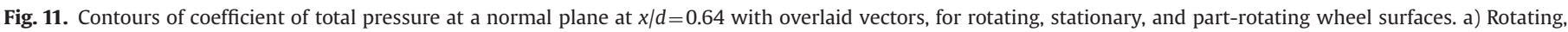
$\mathrm{x} / \mathrm{d}=0.64, \mathrm{~b})$ Tap rotating, $\mathrm{x} / \mathrm{d}=0.64, \mathrm{c})$ Bottom Rotating, $\mathrm{x} / \mathrm{d}=0.64, \mathrm{~d}$ ) and Stationary, $\mathrm{x} / \mathrm{d}=0.64$.

a

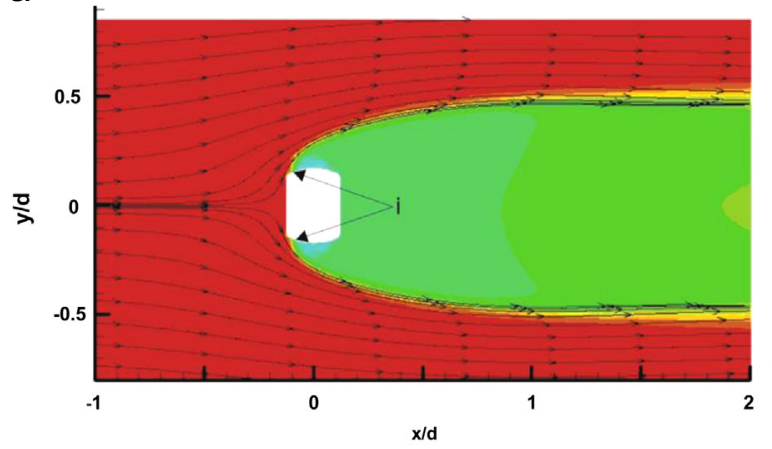

C

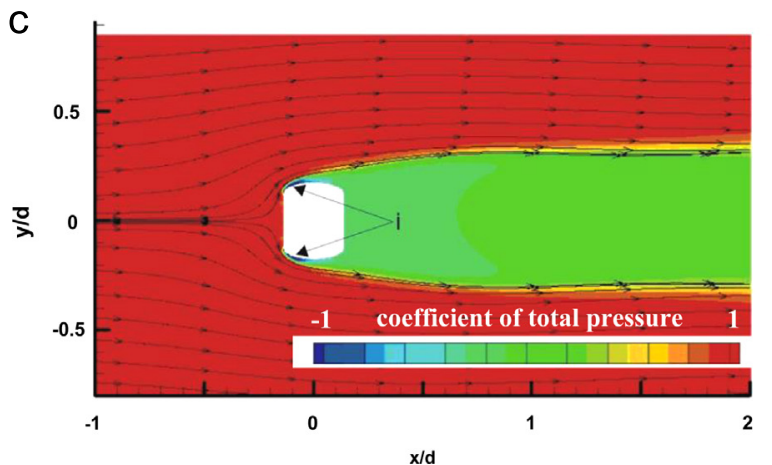

b

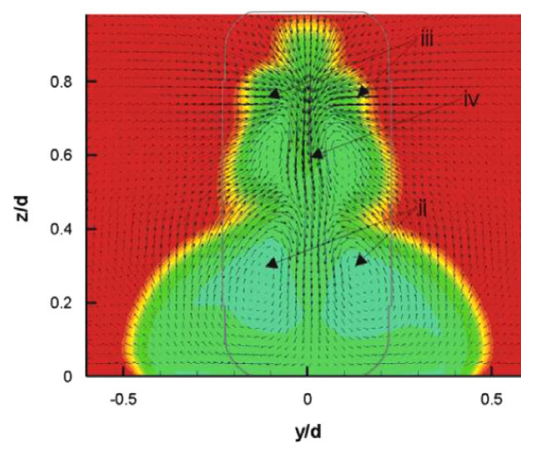

d

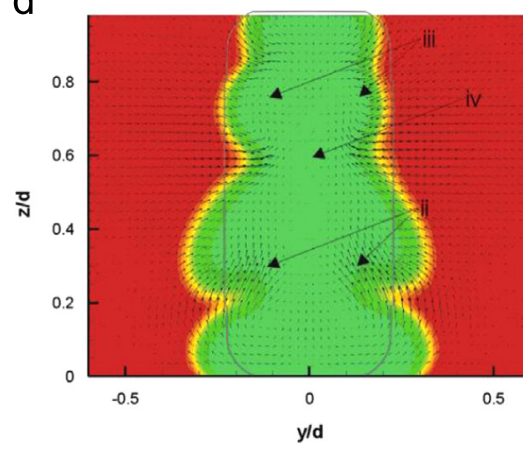

Fig. 12. Contours of coefficient of total pressure at a normal plane at $x / d=0.64$ and on the ground plane, with overlaid vectors, for contact patch step heights of $0.0028 d$ and 0.0085 d. a) $\mathrm{z} / \mathrm{d}=0.015$, step height $=0.0028 \mathrm{~d} \mathrm{~b}) \mathrm{x} / \mathrm{d}=0.64$, step height $=0.0028 \mathrm{~d}$ c) $\mathrm{z} / \mathrm{d}=0.015$, step height $=0.0085 \mathrm{~d}$ d) $\mathrm{x} / \mathrm{d}=0.64$, step height $=0.0085 \mathrm{~d}$. 
pneumatic tyre. A deformable tyre in contact with the ground creates a contact patch instead of contact line, and the size depends on the stiffness characteristics and the load of the tyre. Therefore, it may also be considered that varying the size of the step would give some indication of the dependency of the aerodynamic characteristics on the overall size of the contact patch.

Increasing the step height resulted in the wheel wake reducing in width and increasing in height due to the separation point changing; as the step height was increased, this separation point moved towards the rear of the wheel with a reduction in the lateral departure angle of the jetting flow from the front contact patch (Fig. 12a and c). This is likely to be due to the variation in the contact patch's aspect ratio. Increasing the height of the step increases the length of the contact patch more than its width, resulting in the side portions of the step becoming more perpendicular (Fig. 12c). This result is consistent with Fackrell's (1975) explanations on how the main wheel vortices form-the separation caused by the jetting action at each side and forward of the contact patch is responsible for the main wheel vortices, and here it has been demonstrated that when this separation is delayed or reduced by increasing the height of the step surrounding the contact patch, the strength and size of the main wheel vortices is also reduced (Fig. 12d).

Two other variations are also evident as a result of the change in step height. The upper wheel wake was found to increase in height (Fig. 12d) while the downwash in the centre wheel wake reduced in magnitude. These variations have been previously associated with a forward shift in the separation point from the upper wheel tread region. During the preceding wake study, it was demonstrated that adjusting the wheel tread separation point influences the location of the main wheel vortices. The results presented here suggest that the wheel tread separation point can also be influenced by the primary wheel vortices.

The strength of the primary wheel vortices is responsible for the downwash reduction in the central wheel wake region for a rotating wheel. By reducing the strength, as has been achieved by increasing the size of the step at the contact patch, the central downwash is also reduced and for this reason the top wheel tread separation point also shifts forward as a result.

As the separation point moves further forward on the top of the wheel tread, the drag of the wheel also reduces. At the largest step height tested $(0.0085 d)$ the drag was approximately $20 \%$ less than that obtained with the original step height of $0.0028 d$. As the step height increased, the drag coefficient converged towards a value of approximately 0.4 ; if the step height were reduced it converged to a value of approximately 0.5 (Fig. 13).

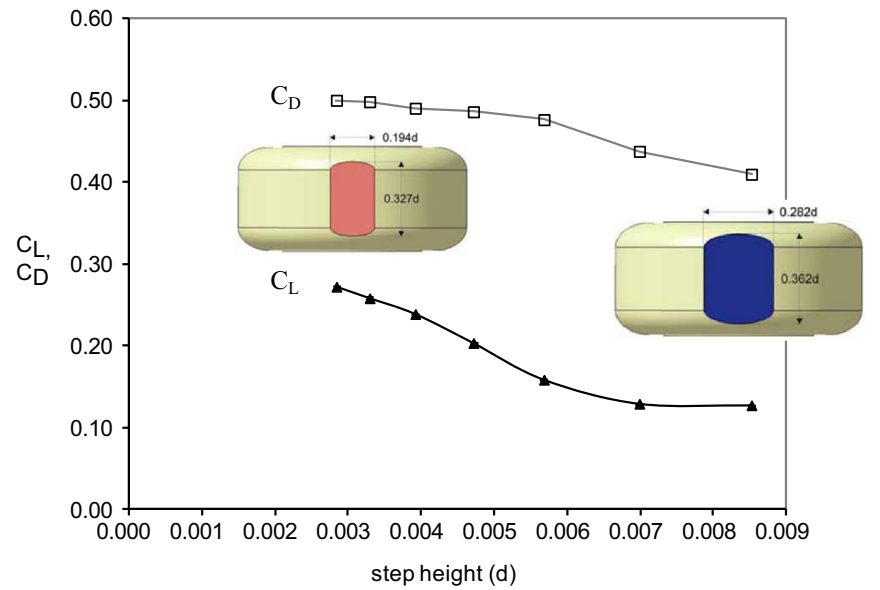

Fig. 13. Lift and drag coefficients vs. wheel contact patch step height.
The lift coefficient also converges towards a value on each extreme of the range tested (Fig. 13). When the separation point moves forward, the peak low pressure coefficient is reduced, causing the lift to be lessened. Lift also proves to be more sensitive to variations in the separation point with the largest step height resulting in a lift reduction of $50 \%$ over the original step height, further confirming the need to keep the step height as small, and therefore as close to the experiment, as possible for the given meshing technique.

Extrapolating from these results, it could be expected that if solid wheels are used during wind tunnel testing of a road vehicle that uses pneumatic tyres, this simplification could contribute to correlation issues between the wind tunnel model and the full scale vehicle. To achieve a realistic wind tunnel model, a tyre that can be deformed in the wind tunnel to reproduce the same contact patch and side walls as that of the tyre on a full scale vehicle would be recommended.

\section{The influence of wheel hub and shoulder geometry}

The pressure acting on the wheel hubs may make small contributions to the lift and drag of the wheel, yet the additional complexity of the hubs presents a challenge to meshing, particularly if fully-structured, and, intuitively, the hubs will also affect the wake flow. The wheel hubs were removed from the A2 wheel geometry, retaining aspect ratio and frontal area. To investigate the influence of shoulder geometry, the existing profile was replaced with a constant radius (Fig. 2). From here on this is referred to as the W1 wheel.

Lift and drag results (Table 2) indicate that the removal of the hubs had a notable effect on the primary force coefficients; a reduction of $20 \%$ and $6 \%$ respectively. Changing the wheel shoulder was found to produce more flow sensitivity than the removal of the hubs. Comparing the results to the A2 wheel with no hubs, in order to isolate the effect that the shoulder has, a $33 \%$ and $25 \%$ increase in lift and drag respectively was obtained for the rotating wheel.

Removing the hubs from the A2 wheel geometry shifted the separation point approximately $5^{\circ}$ forward and reduced the peak pressure obtained over the top of the wheel. The positive pressure gradient $\left(360-285^{\circ}\right)$ produced by these two wheels ahead of their respective peak pressure locations was very similar suggesting that the earlier separation point is also responsible for the reduced peak pressure value obtained in this region. The reduced flow speed and the increased separation from the top surface explain the reduction in the lift.

A unique feature observed for the A2 wheel without wheel hubs was the formation of two additional vortex structures in the wake that are located at approximately $z / d=0.55 d$ (Fig. 14) which may also explain the offset obtained in the central pressure coefficients between the angles of $180^{\circ}$ and $210^{\circ}$ for the two flat sided wheels and the original A2 wheel geometry. This location corresponds with the height from the ground at which these vortices would pass. They are significantly smaller than that of the other four vortices since they are formed by a shear interaction with wheel's side walls while the four others are formed by larger

Table 2

$C_{\mathrm{L}}$ and $C_{\mathrm{D}}$ for rotating wheels with and without hubs and with a constant radius shoulder.

\begin{tabular}{llll}
\hline & Rotating A2 & Rotating A2 (no hubs) & Rotating W1 (constant radius) \\
\hline$C_{\mathrm{L}}$ & 0.28 & 0.20 & 0.37 \\
$C_{\mathrm{D}}$ & 0.51 & 0.47 & 0.51 \\
\hline
\end{tabular}


a

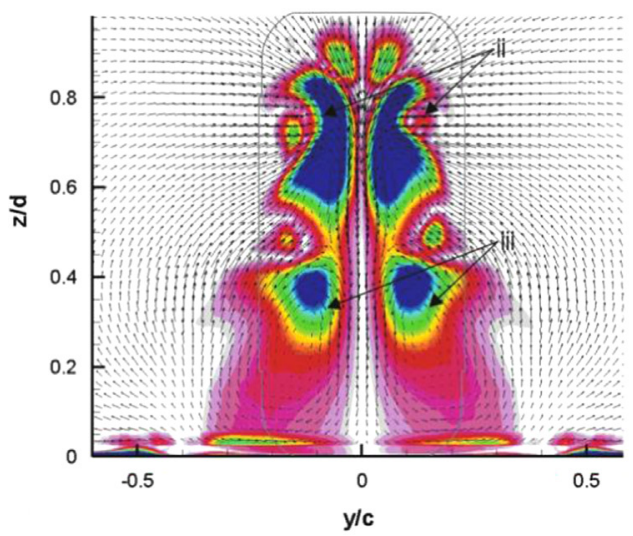

C

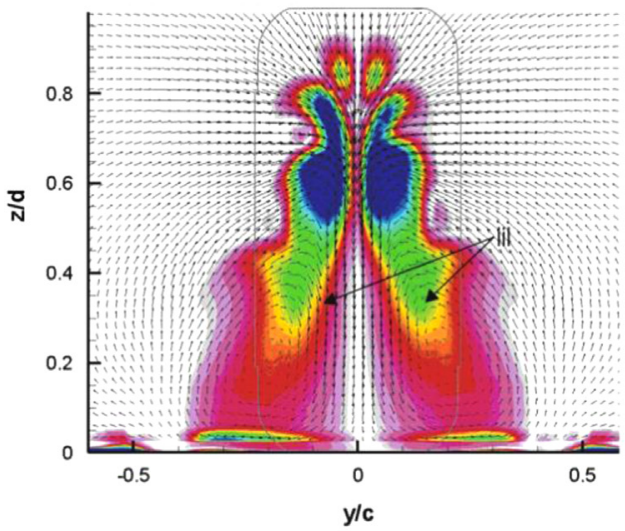

b

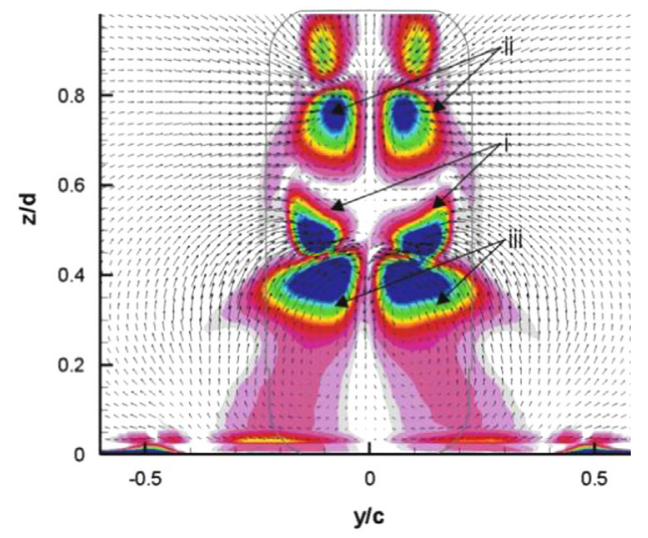

d

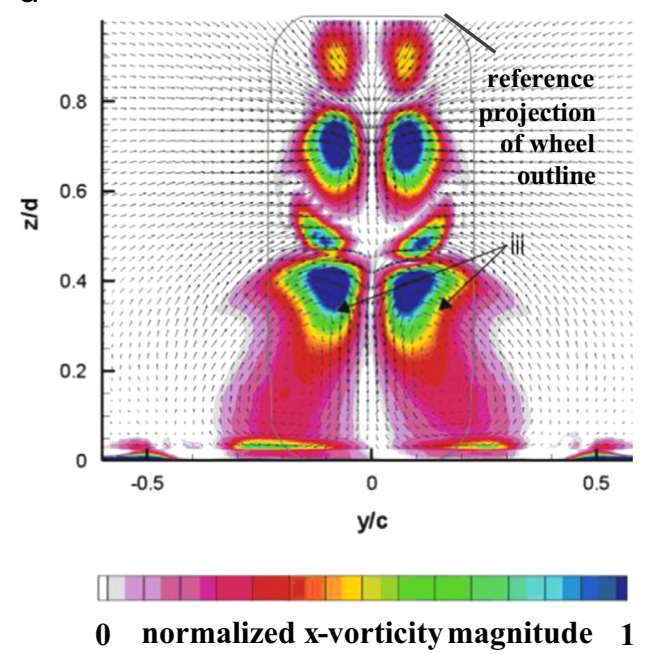

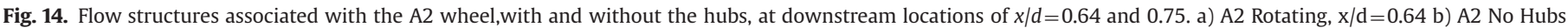
Rotating, $x / d=0.64$ c) A2 Rotating, $x / d=0.75$ d) A2 No Hubs Rotating, $x / d=0.75$.

pressure variations that occur in the wheel wake due to separation. As a result, these two vortices dissipate by the $x / d=0.75$ plane leaving the four initial vortices commonly reported further downstream; this effect was originally speculated to occur by Cogotti (1978).

While McManus and Zhang (2006) and Fackrell (1975) found no evidence of a third pair of vortices in the wheel wake, the results here suggest that this could simply be because their research was conducted with hubs and not with flat sided wheels.

Consistent with the observations linking the separation point with the wake width and height, the A2 wheel without hubs produced a narrower and higher wake in comparison to the original geometry. This smaller wake structure was expected given the reduction in total drag already reported for this configuration. The formation of the two middle vortices increased the entrainment from the sides in the lower wheel wake region and reduced the downwash behind the wheel caused by the earlier separation point from the top surface.

Examining the constant-radius shoulder case, where the overall width was maintained but the tread was effectively wider, a larger region of increased static pressure was observed forward of the wheel that is responsible for the steeper pressure gradient that shown in the central pressure coefficients (360-285'), and would also be expected to contribute to the drag increase obtained by this wheel geometry.

A wider and lower wheel wake was also observed in this instance. The wider wheel tread results in a more extensive contact patch and therefore the jetting action for this wheel occurs further from the wheel centre line than that which resulted previously from the A2 wheel without hubs (Fig. 15). This forces the lower wheel vortices to form further outboard in the wheel wake and this influences the separation point located on the upper portion of the wheel tread. In the middle of the upper wheel wake region, a larger downwash component was obtained which is caused by an increased flow over the top surface of the wheel and a reduced entrainment from the sides. This variation assists with delaying the separation point, and is also responsible for the $85 \%$ increase in lift obtained by changing the wheel shoulder relative to the original.

Despite these large variations in the flow around the wheel, the basic flow structures associated with a rotating wheel were maintained. The two upper wheel vortices appear to have increased in strength, and this may be due to the increased entrainment experienced in the upper central wake region and the steeper adverse pressure gradient experienced by the flow when passing around the rearward portion of the wheel shoulder.

\section{Conclusions}

Several common modelling assumptions and simplifications with regards to isolated wheels have been examined to determine their influence on the flowfields obtained. Geometric variations can alter the details in flow of either a rotating or stationary wheel 
a
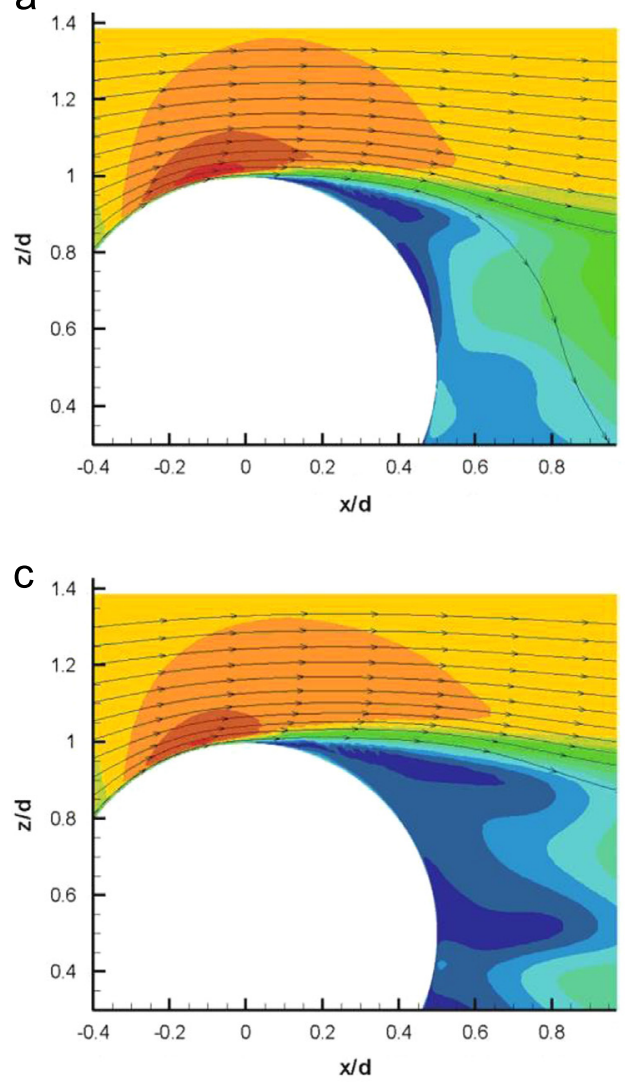

e

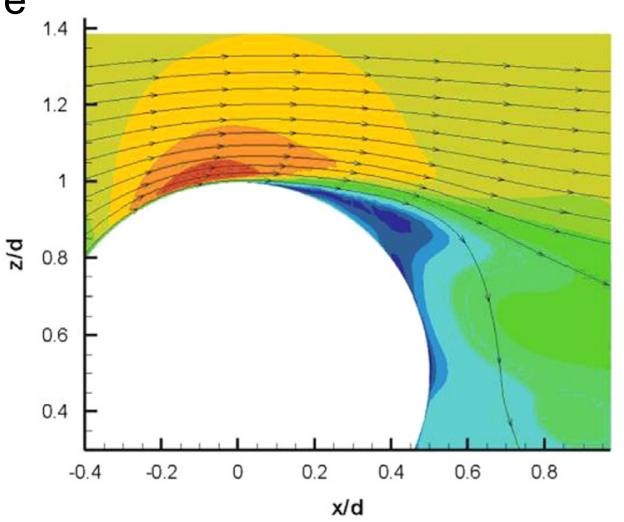

$-1 \quad$ coefficient of total pressure 1

b

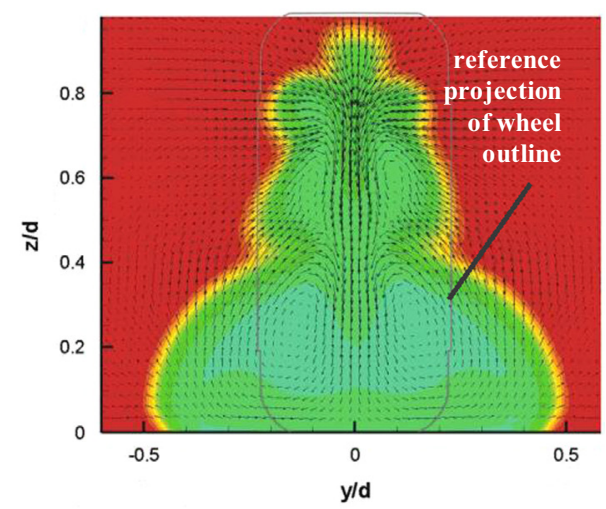

d

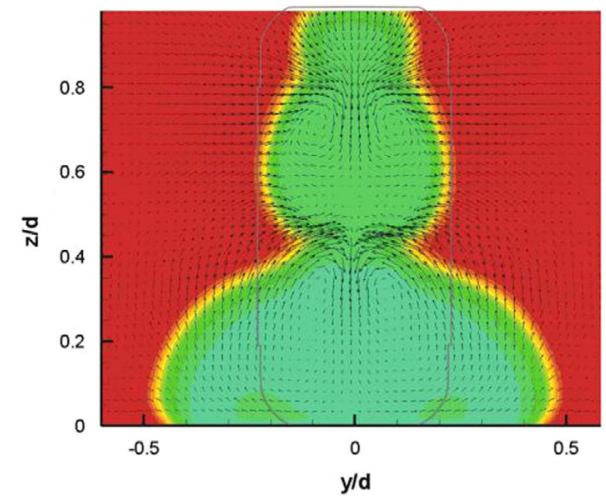

f

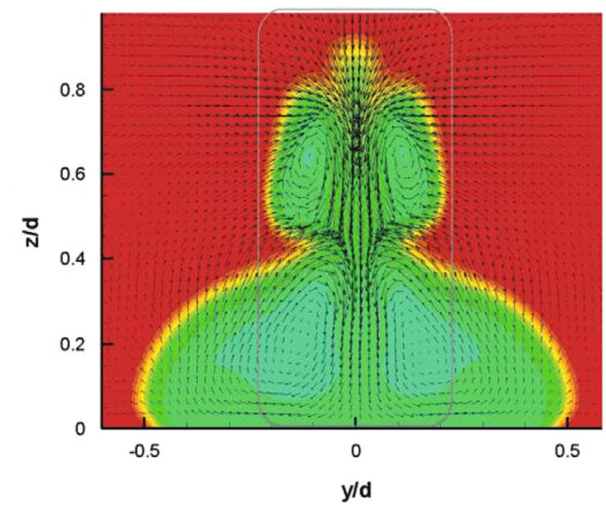

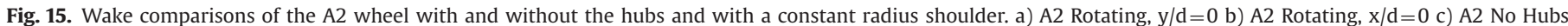
Rotating, $y / d=0$ d) A2 No Hubs Rotating, $x / d=0$ e) W1 Rotating, $y / d=0$ f) $W 1$ Rotating, $x / d=0$.

and as a result create significant changes to the final lift and drag values obtained for a wheel. In comparing rotating to stationary wheels, the main wheel vortices form in a higher and more central position because of the earlier separation caused by the wheel rotation. This causes a reduction of flow entrainment from the top removing the downwash from the central wake region associated with a stationary wheel, and is replaced by an increase in entrainment from the sides for a rotating wheel. The lift and drag have also been shown to be sensitive to the separation location with a more forward separation point causing both the lift and drag to decrease. Only the drag was found to be sensitive to the jetting action at the contact patch. Removing the wheel hubs proved to be influential with regards to predicted lift (30\% less), but less so in terms of drag (8\%), despite a significantly altered wake structure. Equally influential was the imposition of a constant-radius wheel shoulder, which increased the lift by $30 \%$. Relatively small simplifications such as these can therefore exert a large influence on the results, and one can imagine the influence on bodywork downstream for simulations of full vehicles, for instance.

In general it has been re-enforced that, if one is comparing numerical to wind tunnel tests, the more exact the match to the experimental conditions the better the correlation. However, the issue of accurately modelling the contact patch region is not easily 
addressed in conventional CFD and the limitations of this should be considered in comparisons.

\section{Acknowledgements}

The input of the late Professor Eddie Leonardi in supporting this work is gratefully acknowledged.

\section{References}

Axerio-Cilies, J., Iaccarino, G., 2012. An Aerodynamic Investigation of an Isolated Rotating Formula 1 Wheel Assembly. J. Fluids Eng. 134 (12), 1-16.

Axon, L., Garry, K., Howell, J., 1998. An Evaluation of CFD for Modelling the Flow Around Stationary and Rotating Wheels. SAE 980032.

Barber, T.J., Leonardi, E., Archer, R.D., 2002. Causes for discrepancies in ground effect analyses. Aeronaut. J. 106 (1066), 653-657.

Bearman, P.W., De Beer, D., Hamidy, E., Harvey, J.K., 1988. The effect of a moving floor on wind tunnel simulation of road vehicles. SAE, 880245.

Burgin, K., Adey, P.C., Beatham, J.P., 1986. Wind tunnel tests on road vehicle models using a moving belt simulation of ground effect. J. Wind Eng. Ind. Aerodyn. 22 (2), 227-236

Cogotti, A., 1983. Aerodynamic characteristics of car wheels. Int. J. Veh. Des., 173-196 (no. SP 3)

Cruickshank, M., Doig, G., 2014. Application of kriging to motorsport aerodynamic analysis. Appl. Mech. Mater. 553, 217-2122.

Dassanayake, P.R.K., Ramachandran, D., Salati, L., Barber, T.J., Doig, G.C., 2012. Unsteady computational simulation of the flow structure of an isolated wheel in contact with the ground. In: Proceedings of the 18th Australasian Fluid Mechanics Conference, Launceston, Australia, 3-7 Dec.

Diasinos, S., Doig, G., Barber, T.J., 2014. On the interaction of a racing car front wing and exposed wheel. Aeronaut. J. 118, 1210.

Doig, G., Barber, T., Neely, A., 2011. The influence of compressibility on the aerodynamics of an inverted wing in ground effect. J. Fluids Eng.: ASME, 133; , pp. 1-12.

Doig, G., 2014. Transonic and supersonic ground effect aerodynamics. Prog. Aerosp. Sci. 69, 1-28.
Fackrell, J.E., Harvey, J.K., 1973. The flowfield and pressure distributon of an isolated road wheel. In: Advances in Road Vehicle Aerodynamics.

Fackrell, J.E., Harvey, J.K., 1975. The Aerodynamics of an Isolated Road Wheel. In: Proceedings of the 2nd Symposium on Aerodynamic of Sports and Competition Automobiles, AIAA.

Fackrell, J.E., 1975b. The Aerodynamics of an Isolated Wheel Rotating in Contact with the Ground (Ph.D. thesis). Imperial College of Science and Technology, London.

Issakhanian, E., Elkins, C.J., Lo, K. Pong, Eaton, J.K., 2010. An experimental study of the flow around a formula one racing car tire. J. Fluids Eng. 132 (7), 1-8.

Keogh, J., Diasinos, S., Doig, G., 2014. Flow compressibility effects around an openwheel racing car. Aeronautical J. 118 (1210).

Kim, M.S., Geropp, D., 1998. Experimental Investigation of the Ground Effect on the Flow Around Some Two Dimensional Bluff Bodies with Ground Effect with the moving-belt technique. J. Wind Eng. Ind. Aerodyn. 74-76, 511-519.

Knowles, R.D., Saddington, A., Knowles, K., 2002. On the Near Wake of Rotating, 40\% Scale Champ Car Wheels. SAE 2002-01-3293.

Lajos, T., Preszler, L., Finta, L., 1986. Effect of moving ground simulation on the flow past bus models. J. Wind Eng. Ind. Aerodyn. 22 (2), 271-277.

McManus, J., Zhang, X., 2006. A computational study of the flow around an isolated wheel in contact with ground. J. Fluids Eng. 128 (3), 520-530.

Menter, F.R., 1994. Two-equation eddy-viscosity turbulence models for engineering applications. AIAA J. 32 (8), 69-289.

Morelli, A., 1969. Aerodynamic Effects of an Automobile Wheel. MIRA Translation number 47/69 of A.T.A, vol. 22, 6, pp. 81-288.

Pirozzoli, S., Orlandi, P., Bernardini, M., 2012. The fluid dynamics of rolling wheels at low reynolds numbers. J. Fluid Mech. 706, 496-533.

Saddington, A.J., Knowles, R.D., Knowles, K., 2007. Laser Doppler anemometry measurements in the near-wake of an isolated Formula One wheel. Exp. Fluids 42, 671-681.

Shih, T.H., Liou, W.W., Shabir, A., Yang, Z., Zhu, J., 1995. A new k-e Eddy viscocity model for high reynolds number turbulent flows. Comput. Fluids 24 (3), 227-238.

Spalart, P.R. Allmaras, S.R., 1992. A one-equation turbulence model for aerodynamic flows. In: Proceedings of 30th AIAA Aerospace Sciences Meeting and Exhibit, Reno, NV, p. 1992

Stapleford, W.R., Carr, G.W., 1970. Aerodynamic Characteristics of Exposed Rotating Wheels (MIRA Technical Report 1970/2). 